\title{
THE PERSISTENCE OF LOGCONCAVITY FOR POSITIVE SOLUTIONS OF THE ONE DIMENSIONAL HEAT EQUATION
}

\author{
G. KEADY
}

(Received 29 January 1988; revised 30 December 1988)

Communicated by A. J. Pryde

\begin{abstract}
Consider positive solutions of the one dimensional heat equation. The space variable $x$ lies in $(-a, a)$ : the time variable $t$ in $(0, \infty)$. When the solution $u$ satisfies $(i) u( \pm a, t)=0$, and (ii) $u(\cdot, 0)$ is logconcave, we give a new proof based on the Maximum Principle, that, for any fixed $t>0, u(\cdot, t)$ remains logconcave. The same proof techniques are used to establish several new results related to this, including results concerning joint concavity in $(x, t)$ similar to those considered in Kennington [15].
\end{abstract}

1980 Mathematics subject classification (Amer. Math. Soc.) (1985 Revision): 35 K 05.

\section{Introduction}

1.1. First results. Section 1 states our main results. They are proved in Sections 4, 5 and 6. In the intervening Sections 2 and 3 some standard results, needed for the new proofs, are collected together.

Let $\Omega \subset \mathbf{R}^{d}$ be an open bounded convex set. Except in those parts of Section 1 where we review results published elsewhere, $d=1$. A real function $f$ defined over a convex set $\Omega$ is called logconcave if $f$ is a nonnegative function on $\Omega$ and if, with

$$
\begin{gathered}
x_{\tau}=(1-\tau) x_{0}+\tau x_{1}, \\
f\left(x_{\tau}\right) \geq\left(f\left(x_{0}\right)\right)^{1-\tau}\left(f\left(x_{1}\right)\right)^{\tau} \quad \forall x_{0}, x_{1} \in \Omega, \tau \in[0,1] .
\end{gathered}
$$

(C) 1990 Australian Mathematical Society 0263-6115/90\$A2.00+0.00 
Similarly, a real function $f$ defined over a convex set $\Omega$ is called logconvex if $f$ is a positive function on $\Omega$ and $1 / f$ is logconcave.

Consider solutions to the heat equation: $u=u(x, t)$ defined for $x \in \bar{\Omega}$ and for $t \geq 0$ satisfying

$$
\begin{aligned}
\frac{\partial u}{\partial t} & =\Delta u \quad \text { in } \Omega \times(0, \infty), \\
u & =0 \quad \text { on } \partial \Omega \times[0, \infty), \\
u(x, 0) & =u_{0}(x) \quad \forall x \in \Omega .
\end{aligned}
$$

where $u_{0}$ is a given function with $u_{0}$ vanishing on $\partial \Omega$.

The concavity properties of solutions of problems similar to problem (P) have been considered in many papers, including [3], [10], [11], [16] and, most recently, Kennington [15]. Theorem 1.3, proved in Section 6 below is suggested by Kennington's results, though our methods of proof are different from Kennington's.

Our methods of proof can also be used to give new proofs, when $d=1$, of older results, for example Theorem 1.0, which we now state.

THEOREM $1.0(d \geq 1)$. Let $\Omega$ be a convex open subset of $\mathbf{R}^{d}$ and let $u_{0}$, the initial distribution of temperature on $\Omega$, be positive and logconcave.

Then the distribution of temperature $u(z, t)$, solving problem $(\mathbf{P})$, at any later time $t$ is logconcave.

The one dimensional version which is stated below, and some generalisations, are the main results which will be proved in this paper. A function $f \in C^{2}(-a, a)$ which is positive in $(-a, a)$ is logconcave if and only if $(\log f)_{x x} \leq 0$ in $(-a, a)$ : it is strictly logconcave if and only if $(\log f)_{x x}<0$ in $(-a, a)$. With the same restrictions of smoothness and positivity of $f$,

$$
(\log f)_{x x}=\frac{f f_{x x}-f_{x}^{2}}{f^{2}}
$$

TheOREM 1.0. Let $\Omega=(-a, a) \subset \mathbf{R}$ and let $u_{0}$, the initial distribution of temperature on $(-a, a)$, satisfy

(i) $u_{0} \in C^{\infty}[-a, a], u_{0}$ and all even-order derivatives are zero at $x= \pm a$,

(ii) in $(-a, a)$ the function $u_{0}$ is (strictly) positive, $u_{0}>0$, and strictly logconcave,

(iii) $\sup _{[-a, a]}\left|\frac{u_{0}^{\prime \prime}}{u_{0}}\right|<\infty$.

Let $u \in C^{\infty}$ solve problem $(\mathrm{P})$. Then the distribution of temperature $u(x, t)$, at any later time $t$ is a strictly logconcave function of $x$.

The proof is given in Section 4. 
We remark that the smoothness hypotheses above are stronger than necessary for the result. A limiting argument, not given in this paper, can be used to weaken them, and to establish the $d=1$ version of Theorem $1.0(d \geq 1)$.

Define

$$
u_{1}(x, t)=\exp \left(-\frac{\pi^{2} t}{4 a^{2}}\right) \cos \frac{\pi x}{2 a} .
$$

The function $u_{1}(., t)$ is, at each fixed $t$ concave in the space variable, and

$$
u_{1} u_{1 x x}-u_{1 x}^{2}=-\frac{\pi^{2}}{4 a^{2}} \exp \left(-\frac{\pi^{2} t}{2 a^{2}}\right)<0 .
$$

Returning to positive solutions of problem $(\mathrm{P})$, since

$$
u(x, t) \sim c u_{1}(x, t) \text { as } t \rightarrow \infty,
$$

with

$$
c=\frac{1}{a} \int_{-a}^{a} u_{0}(\hat{x}) \cos \frac{\pi \hat{x}}{2 a} d \hat{x}
$$

we have

$$
\frac{u u_{x x}-u_{x}^{2}}{u_{1} u_{1 x x}-u_{1 x}^{2}} \rightarrow c^{2} \quad \text { as } t \rightarrow \infty
$$

and so $u$ is logconcave at large time. For the proof, see Lemma 3.5. When it is convenient to use this in our new proofs it will be used.

Relaxing the condition that $\Omega$ be bounded, for this paragraph take $\Omega=\mathbf{R}$. Define

$$
K_{\infty}(\hat{x} ; x, t)=\frac{1}{\sqrt{4 \pi t}} \exp \left(-\frac{(x-\hat{x})^{2}}{4 t}\right) .
$$

At each fixed value of $\hat{x}, K_{\infty}$ is a solution of the heat equation. Further, at each fixed value of $t, K_{\infty}$ is logconcave in $x$. This provides an example to check against Theorem 1.0. The function $K_{\infty}$ is the kernel function for the heat equation on $\mathbf{R}$.

We remark that the logconcavity of the kernel function for other convex domains was the route through to the first proof of Theorem 1.0, which was given by Brascamp and Lieb [3]. Korevaar [16] gave a different proof. Both these methods of proof, (and some others, including one based on Burger's equation) are given in a survey in Keady [13]. Other surveys are given in Kawohl [10], [11], and Ellis and Newman [5]. The survey [13] also indicates some of the ways that the proofs given in this paper can be adapted to new problems, not just the heat equation problem treated in this paper. Our method of proof, which we give in Section 4, can be modified to prove various other results. These results are Theorems 1.2, 4.1 (and Theorem A.1.0, applying to the space discretisation of the heat problem (P), given in Keady [13]), all of which appear to be new, and Theorem 1.3. Our methods 
of proof are similar to those used, for elliptic problems, in Makar-Limanov [17], Acker, Payne and Philippin [1], Keady [12] and Caffarelli and Friedman [4].

As stated earlier, Theorem 1.0 is true for $\Omega \subset \mathbf{R}^{d}$ when $d>1$ as well as $d=1$. Theorem 1.1(i) below is a very elementary result which is true only for $d=1$ as it relies on the coincidence of superharmonic and concave function classes in $\mathbf{R}^{\mathbf{1}}$. (For a counterexample in $d=2$ consider the large time asymptotics when $\Omega$ is a disk.) A more difficult result is Theorem 1.1 (ii) which also has only been proved when $d=1$. Theorem 1.1(ii) concerns quasiconcave functions. A nonnegative function $f$ defined on a convex set $\Omega$ is said to be quasiconcave if $\{x \mid f(x)>c\}$ is convex for all positive $c$.

TheOREM 1.1. Let $\Omega=(-a, a) \subset \mathbf{R}^{1}$. Let $u$ solve problem $(\mathrm{P})$.

(i) Let $u_{0}$ be concave. Then $u(x, t)$, at any later time $t$, is a concave function of $x$.

(ii) If $u_{0}$ is positive and quasiconcave, a characterisation for which is

$$
u_{0 x} \geq 0 \text { for }-a<x<\xi \text { and } u_{0 x} \leq 0 \text { for } \xi<x<a \text {, }
$$

then so is $u(\cdot, t)$ at any subsequent time $t$.

Proof. (i) We first prove the result when we have more regularity: namely where $u_{0} \in C^{\infty}([-a, a])$ with $u_{0}$ and all even-order derivatives zero at $x=$ $\pm a$. (The general situation with less regularity on $u_{0}$ can be obtained from this particular situation by a limiting procedure.) Thus we consider such smooth $u_{0}$, and suppose that $u_{0}$ is not identically zero. Since $u_{0}$ is concave, $u_{t}(x, 0)=u_{x x}(x, 0) \leq 0$. Next the function $u_{t}$ satisfies

$$
\begin{aligned}
\left(u_{t}\right)_{t} & =\left(u_{t}\right)_{x x} \quad \text { in } \Omega \times(0, \infty), \\
u_{t} & =0 \quad \text { on } \partial \Omega \times[0, \infty), \\
u_{t}(x, 0) & \leq 0 \quad \forall x \in \Omega .
\end{aligned}
$$

Thus, by the Maximum Principle,

$$
u_{t}<0 \quad \text { in } \Omega \times(0, \infty) \text {. }
$$

Hence $u_{x x}(x, t)<0$ in $\Omega \times(0, \infty)$ so that, at fixed $t>0, u(\cdot, t)$ is strictly concave.

(ii) The maximum principle proof of this is complicated and is omitted here. See Matano [18], Nickel [19]. The sketch of a proof in Keady [13] requires the additional hypothesis that $u_{0}$ be such that $u$ is (real-)analytic in a domain containing $\Omega \times[0, \infty)$ in order to apply level curve arguments. Polya [20] gives a different proof. 
The new proof of Theorem 1.0 given in Section 4 is similar to the elementary proof of Theorem 1.1(i) in that both use the Maximum Principle.

(For the definition of when a nonnegative function $f$ defined over a convex set $\Omega$ is called $\alpha$-concave see [3] or [13]. Theorem 1.1 establishes the persistence of $\alpha$-concavity when $\alpha=1$ and when $\alpha=-\infty$, while Theorem 1.0 establishes the persistence when $\alpha=0$. For other values of $\alpha<1$ there are counterexamples: see the corrections to [13]. For the use of different techniques on a different parabolic problem, but still establishing the persistence of power concavity, see Benilan and Vazquez [2].)

Theorem 1.0 generalises in various ways. As one example we mention Theorem 4.1. Theorem 4.1 can be established by the methods of Section 4 but not, it appears, by the methods of the earlier proofs of Theorem 1.0.

1.2. More general boundary data. More general boundary conditions than $u( \pm a, t)=0$ could be considered. We define

$$
I_{2}=u u_{x x}-u_{x}^{2} \text {. }
$$

Indeed our proof of Theorem 1.0 will follow from Theorem 1.2:

THEOREM 1.2. Suppose that $u$ is a solution of the heat equation, is positive in $\Omega \times[0, \infty)$, and is sufficiently smooth. Suppose that $I_{2}$ is known to be nonpositive

(i) initially, that is at $t=0$, and

(ii) in neighbourhoods of the boundaries $x= \pm a$.

Then the distribution of temperature $u(\cdot, t)$ is, at each fixed value of $t>0$, a logconcave function of $x$.

Proof. This is an immediate consequence of Theorem 4.0(a) the proof of which will be given in Section 4 .

1.3. Concavity jointly in $(x, t)$. Kennington [15] has established results suggesting Theorem 1.3 below.

Consider positive functions of the two variables $(x, t)$. When these are suitably smooth these are jointly concave (in $(x, t))$ if the matrix of second partial derivatives is negative semidefinite. A positive function $u(x, t)$, which is suitably smooth, is jointly logconcave if and only if

$$
\begin{gathered}
I_{2}=u u_{x x}-u_{x}^{2} \leq 0, \quad J_{2}=u u_{t t}-u_{t}^{2} \leq 0, \\
K_{2}=\left(u u_{x x}-u_{x}^{2}\right)\left(u u_{t t}-u_{t}^{2}\right)-\left(u u_{x t}-u_{x} u_{t}\right)^{2} \geq 0 .
\end{gathered}
$$

The function $u_{1}$, being a product of a function of $t$ which is logconcave and of a function of $x$ which is (log-)concave, is an example of a function which is jointly logconcave on $(-a, a) \times(0, \infty)$. 
Theorem 1.3. Let $\Omega=(-a, a) \subset \mathbf{R}$. Suppose that $u$ is a solution of the heat equation, is positive and is sufficiently smooth. Suppose that $I_{2}$ and $-K_{2}$ (and hence $J_{2}$ ) are known to be nonpositive

(i) initially, that is at $t=0$, and

(ii) in neighbourhoods of the boundaries $x= \pm a$.

Then the distribution of temperature $u(x, t)$ is a logconcave function of $(x, t)$ in $(-a, a) \times(0, T)$.

Proof. Combine the results of Theorems 1.2 and 6.0. The latter is proved in Section 6.1.

The questions in Section 6.2 (and generalisations in [13]) are of the following form: given that $u_{0}$ is logconcave (in $x$ ) and then further hypotheses on $u_{0}$, what further properties does $u$ have beyond those established by Theorem 1.0. Perhaps the simplest question is Question 6.1: do the level curves of the kernel function $K$ enclose convex sets in $(x, t)$-space?

Another paper with some consideration of joint-concavity questions arising in parabolic problems is Keady and Stakgold [14].

\section{Maximum principles}

The following notation will be used:

$$
\begin{aligned}
& Q_{T}=(a, b) \times(0, T), \\
& S_{T}=([a, b] \times\{0\}) \cup(\{a\} \times[0, T)) \cup(\{b\} \times[0, T)) .
\end{aligned}
$$

The set $S_{T}$ will be called the parabolic boundary of $Q_{T}$.

The following form of the Maximum Principle is a special case of that proved in Protter and Weinberger [21], Theorem 4, p. 172 (where less smoothness on the coefficients is required than that in our hypotheses). An alternative source for the proof, when $A_{1}, A_{0}$ and $f$ are continuous on $\bar{Q}_{T}$ is Friedman [7], Theorem 1, p. 34.

Maximum Principle 2.1. Let $A_{0}, A_{1}$ and $f$ belong to $C\left(\bar{Q}_{T}\right)$. Suppose $u$ is in the function space

$$
u \in C^{1}\left(\bar{Q}_{T}\right), \quad \frac{\partial^{2} u}{\partial x^{2}} \in C\left(\bar{Q}_{T}\right)
$$

and satisfies

$$
L u \equiv \frac{\partial u}{\partial t}-\frac{\partial^{2} u}{\partial x^{2}}+A_{1} \frac{\partial u}{\partial x}+A_{0} u=f \geq 0 \quad \text { in } Q_{T},
$$


where

$$
A_{0} \geq 0 \text { in } Q_{T}
$$

(i) If

$$
m=\inf _{Q_{T}} u, \quad m<0,
$$

and there exists $(\xi, \tau) \in Q_{T}$ such that $u(\xi, \tau)=m$, then $u \equiv m$ in $Q_{\tau}$.

(ii) If $u$ is not identically zero throughout $Q_{T}$ and

$$
u \geq 0 \text { on } S_{T} \text {, }
$$

then

$$
u>0 \text { in } Q_{T} \text {. }
$$

(iii) (The Hopf result.) With the hypotheses in (ii), if $u(b, \tau)=0$ for $0<$ $\tau<T$, then

$$
u_{x}(b, \tau)<0
$$

In Sections 4, 5 and 6 we will need Maximum Principles where we do not know the sign of $A_{0}$. Following Protter and Weinberger [21, p. 175] we have the following.

Maximum Principle 2.2. Suppose that all the hypotheses of Maximum Principle 2.1 are satisfied except for the nonnegativity constraint (2.1).

Then the conclusions (ii), (iii) of Maximum Principle 2.1 remain true.

Proof. Let

$$
L^{(2)} u^{(2)} \geq 0,
$$

satisfy the hypotheses of Maximum Principle 2.2. Let

$$
u^{(1)}=e^{-\gamma t} u^{(2)}, \quad A_{0}^{(1)}=A_{0}^{(2)}+\gamma .
$$

Define the operator $L^{(1)}$ by

$$
e^{+\gamma t} L^{(1)} u^{(1)}=L^{(2)} u^{(2)}
$$

With

$$
\gamma=\sup _{\bar{Q}_{T}}\left|A_{0}^{(2)}\right|,
$$

the nonnegativity constraint (2.1) of Maximum Principle 2.1 on $A_{0}^{(1)}$ is satisfied.

(Similar results are given in Sperb [22, p. 21].) 
Extensions of these Maximum Principles, proved in Gidas, Ni and Nirenberg [8] are used in [4] and [13].

\section{Miscellaneous results for the heat equation}

Various bounds used in the proofs of Theorems $1.0,1.2,1.3$, etc. are given in this section. Detailed proofs of the theorems stated in this section are given in [13].

THEOREM 3.1 (Bounds on $u$ ). Let $u$ solve the heat problem (P) with $u_{0} \in$ $C[-a, a]\left(\right.$ with $\left.u_{0}( \pm a)=0\right)$. Then

$$
M_{n}(t)=\frac{1}{2 a} \int_{-a}^{a} u(x, t)^{2 n} d x, \quad n=1,2, \ldots,
$$

is a monotone decreasing function of $t$ for $t \geq 0$.

Furthermore

$$
\max _{-a \leq x \leq a}|u(x, t)|=\lim _{n \rightarrow \infty} M_{n}(t)^{1 / 2 n},
$$

and, defining

$$
M_{\infty}(t)=\max _{-a \leq x \leq a}|u(x, t)|,
$$

$M_{\infty}$ is a monotone decreasing function of $t$ for $t \geq 0$.

THEOREM 3.2 (Bounds on $\left|u_{x}\right|$ ). Let $u$ solve the heat problem $(\mathrm{P})$ with $u_{0} \in C^{1}[-a, a]$ (with $u_{0}( \pm a)=0$ ). Define

$$
M_{\infty}^{(1)}(t)=\max _{-a \leq x \leq a}\left|u_{x}(x, t)\right| .
$$

Then $M_{\infty}^{(1)}$ is a monotone decreasing function of $t$ for $t \geq 0$.

Engler [6] proves a generalisation of this where the interval $(-a, a) \subset \mathbf{R}$ is replaced by convex $\Omega \subset \mathbf{R}^{d}$, and $\left|u_{x}\right|$ is replaced by $|\nabla u|$.

THEOREM 3.3 (Bounds on $I_{2}$ ). Let $u$ solve the heat problem $(\mathrm{P})$ with $u_{0}$ satisfying hypothesis (i) of Theorem 1.0. Then $u, u_{x x}, u_{x}^{2}$ and $I_{2}=u u_{x x}-u_{x}^{2}$, are bounded on $[-a, a] \times[0, \infty)$.

Proof. We have already seen that

$$
0 \leq u \leq \max _{(-a, a)} u_{0} \text { on }(-a, a) \times(0, \infty),
$$

and 


$$
0 \leq u_{x}^{2} \leq \max _{[-a, a]} u_{0 x}^{2} \text { on }(-a, a) \times(0, \infty) .
$$

All that remains to do is to bound $u_{t}=u_{x x}$. We have

$$
\begin{array}{ll}
\left(u_{x x}\right)_{t}=\left(u_{x x}\right)_{x x} & \text { in }(-a, a) \times(0, \infty) \\
u_{x x}( \pm a, t)=0 & \text { for all } t \in(0, \infty) \\
u_{x x}(x, 0)=u_{0 x x} & \text { for all } x \in(-a, a)
\end{array}
$$

Thus

$$
\min \left\{0, \min _{[-a, a]}\left(u_{0 x x}\right)\right\} \leq u_{x x}(x, t) \leq \max \left\{0, \max _{[-a, a]}\left(u_{0 x x}\right)\right\} \quad \text { in }(-a, a) \times(0, \infty) \text {. }
$$

This completes the proof.

THEOREM 3.4 (Bounds on $u_{t} / u$ ). Let $u$ solve the heat problem $(\mathrm{P})$ with the initial data $u_{0}$ satisfying hypotheses (i) and (iii) of Theorem 1.0 and $u_{0}>0$ in $(-a, a)$, with

$$
\beta_{-} \leq \frac{u_{0 x x}(x)}{u_{0}(x)} \leq \beta_{+} \text {for } x \in(-a, a) .
$$

Then

$$
\beta_{-} \leq \frac{u_{t}}{u} \leq \beta_{+} \text {on }(-a, a) \times[0, \infty)
$$

Proof. We have, with $v=u_{t}-\beta u$, that $v$ satisfies the heat equation, that $v( \pm a, t)=0$ for all $t$ and, that, initially,

$$
\left(\beta_{-}-\beta\right) u_{0} \leq v(x, 0) \leq\left(\beta_{+}-\beta\right) u_{0} .
$$

With $\beta$ set to $\beta_{-}$, the Maximum Principle 2.1 gives $u_{t}-\beta_{-} u \geq 0$ on $(-a, a) \times$ $[0, \infty)$. Similarly, with $\beta$ set to $\beta_{+}$, the Maximum Principle 2.1 gives $u_{t}-$ $\beta_{+} u \leq 0$ on $(-a, a) \times[0, \infty)$.

LEMMA 3.5 (Asymptotics for $I_{2}$ ). Let $u$ be a positive solution of the heat problem (P) with the initial data $u_{0}$ satisfying hypothesis (i) of Theorem 1.0. Let $c$ be defined by equation (1.1b). Then

$$
\frac{u u_{x x}-u_{x}^{2}}{u_{1} u_{1 x x}-u_{1 x}^{2}} \rightarrow c^{2} \quad \text { as } t \rightarrow \infty,
$$

where the convergence is uniform in $x$.

Proof. Write $u=c u_{1}+R$ where

$$
R(x, t)=\exp \left(-\frac{\pi^{2} t}{a^{2}}\right) \sum_{j=2}^{\infty} b_{j} \exp \left(-\frac{\left(j^{2}-4\right) \pi^{2} t}{4 a^{2}}\right) \sin \left(\frac{j \pi(x+a)}{2 a}\right),
$$


where the $b_{j}$ are Fourier coefficients obtained from $u_{0}$. Since $u_{0}$ is smooth, for all $t \geq 0$ the preceding Fourier series for $u$ and the similar Fourier series for its $x$-derivatives are absolutely and uniformly convergent. We have

$$
\frac{u u_{x x}-u_{x}^{2}}{u_{1} u_{1 x x}-u_{1 x}^{2}}=c^{2}+\frac{c u_{1 x x} R+c u_{1} R_{x x}-2 c u_{1 x} R_{x}+R R_{x x}-R_{x}^{2}}{u_{1} u_{1 x x}-u_{1 x}^{2}} .
$$

The second term is order $\exp \left(-\left(3 \pi^{2} t\right) /\left(4 a^{2}\right)\right)$, and the result follows.

THEOREM 3.6 (A bound on $I_{2}$ at the boundary). Let $u$ solve the heat problem $(\mathrm{P})$ with $u_{0}$ satisfying all the hypotheses of Theorem 1.0 , and in addition part (ii) strengthened so that with

$$
I_{2}=u u_{x x}-u_{x}^{2}
$$

on $[-a, a], I_{2}$ is strictly negative initially. Then there exists $\delta$, independent of $t$, such that

$$
I_{2}<0 \text { for }-a \leq x \leq-a+\delta \text { and for } a-\delta \leq x \leq a .
$$

Proof. The Hopf form of the Maximum Principle 2.1 gives that

$$
I_{2}<0 \text { at } x= \pm a, t>0,
$$

and so there exists a positive function $\delta(t)$ such that

$$
I_{2}<0 \text { for }-a \leq x \leq-a+\delta(t) \text { and } a-\delta(t) \leq x \leq a .
$$

Next we make some estimates on $\delta(t)$.

Using the hypothesis in the theorem and continuity of $I_{2}$ in a neighbourhood of $t=0$ we have that, for some small $\varepsilon>0$,

$$
\delta(t) \geq \delta_{0}>0 \text { for } 0 \leq t \leq \varepsilon .
$$

Next using Lemma 3.5 on the asymptotic behaviour of solutions of problem (P) as $t \rightarrow \infty$, for some large $T<\infty$,

$$
\delta(t) \geq \delta_{\infty}>0 \text { for } T \leq t<\infty .
$$

We could take $\delta_{\infty}=a$.

Using the continuity of $I_{2}$ in $[-a, a] \times[\varepsilon, T]$ we have, from $I_{2}( \pm a, t)<0$, that

$$
\delta(t) \geq \delta_{\text {int }}>0 \text { for } \varepsilon \leq t \leq T .
$$

This establishes the result. 
Remark. The smoothness hypothesis (i) of Theorem 1.0 is much stronger than is needed for Theorems 3.3 to 3.6.

\section{Concavity properties in $x$ at fixed $t$}

The function $(\log u)_{x}$ satisfies Burger's equation,

$$
\left((\log u)_{x}\right)_{t}-\left((\log u)_{x}\right)_{x x}=2(\log u)_{x}\left((\log u)_{x}\right)_{x} .
$$

Differentiating with respect to $x$ gives, with

Defining

$$
\begin{gathered}
I_{0}=(\log u)_{x x}, \\
I_{0 t}-I_{0 x x}-\frac{2 u_{x}}{u} I_{0 x}=2 I_{0}^{2} .
\end{gathered}
$$

we have

$$
I_{k}=u^{k}(\log u)_{x x},
$$

$$
\begin{aligned}
I_{2 t}-I_{2 x x}+\frac{2 u_{x}}{u} I_{2 x} & =\frac{2}{u^{2}}\left(I_{2}+u_{x}^{2}\right) I_{2}=\frac{2 u_{t}}{u} I_{2}, \\
I_{k t}-I_{k x x}+2(k-1) \frac{u_{x}}{u} I_{k x} & =\left(2 u^{-k} I_{k}+k(k-1) \frac{u_{x}^{2}}{u^{2}}\right) I_{k}, \\
& =\frac{1}{u^{2}}\left(2 u u_{t}+\left(k^{2}-k-2\right) u_{x}^{2}\right) I_{k} .
\end{aligned}
$$

The preceding equations are of the form (with $n=1$ )

$$
I_{t}-I_{x x}+A_{1} I_{x}+A_{0} I=0, \quad A_{0}=\sum_{j=0}^{n} a_{j} I^{j} .
$$

If, with some $I=w I_{2}$ with $w>0$, we have

$$
a_{j} \geq 0 \text { for } 0 \leq j \leq n,
$$

the Maximum Principle 2.1, in a straightforward application (except for certain technicalities about unbounded coefficients and boundedness of $I$ ), will give

$$
I(0, x) \leq 0 \text { initially } \Rightarrow I(x, t)<0 \quad \forall t>0 .
$$

With $I=u^{k} I_{2}$ we do not have $a_{j} \geq 0$. (A table of $a_{j}$ for various $W$ with $I=W\left(u, u_{x}\right) I_{2}$ is given in Keady [13].) However it is possible to use Maximum Principle 2.2 as follows. Our proof of Theorem 1.0 (as also that of Theorem 4.1) will follow from a combination of a boundary concavity result and Theorem 1.2. Theorem 1.2 is an immediate consequence of an Interior Concavity Maximum Principle, Theorem 4.0 below. Only part (a) and not part (b) will be needed. 
TheOREM 4.0 (Interior concavity maximum principle). Let $u$ be a positive solution of the heat equation on $\bar{Q}_{T}$.

(a) If $I_{2} \leq 0$ on $S_{T}$ then $I_{2} \leq 0$ on $\bar{Q}_{T}$.

(b) If $I_{2} \geq 0$ on $S_{T}$ then $I_{2} \geq 0$ on $\overline{Q_{T}}$.

Proof. This follows from Maximum Principle 2.2 (and the results of Section 3 in as much as they ensure that the coefficients in the equation (4.1) are bounded as is required by Maximum Principle 2.2).

Proof of Theorem 1.0 with $d=1$. We first prove the result with the stronger hypotheses of Theorem 3.6. From the asymptotic behaviour given in Lemma 3.5 it can be seen that, for $T$ sufficiently large, $u(\cdot, T)$ is strictly logconcave. Thus

$$
I_{2}(x, T)<0 \text { for }-a \leq x \leq a .
$$

Also, by the hypotheses on $u_{0}$

$$
I_{2}(x, 0)<0 \text { for }-a \leq x \leq a .
$$

By Theorem 3.6, for $0 \leq t \leq T$ and some $\varepsilon>0$

$$
I_{2}(x, t)<0 \text { for }-a \leq x<-a+\varepsilon \text { and for } a-\varepsilon<x \leq a,
$$

and also, by Theorem 3.3, $I_{2}$ is bounded on $[-a, a] \times[0, T]$. By Theorems 3.3 and 3.4 the coefficients are also bounded on $[-a+\varepsilon, a-\varepsilon] \times[0, T]$, so that, applying the Interior Concavity Maximum Principle, Theorem 4.0, on $[-a+\varepsilon, a-\varepsilon] \times[0, T]$, establishes the theorem, provided $I_{2}\left(u_{0}\right)>0$ on the closed interval $[-a, a]$.

The hypothesis (ii) of Theorem 1.0 is that $I_{2}\left(u_{0}\right)>0$ on the open interval $(-a, a)$. Such $u_{0}$ may be approximated as close as we wish in the maximum norm by $u_{0}^{(e)}$ with $I_{2}\left(u_{0}^{(\varepsilon)}\right)>0$ on the closed interval $[-a, a]$. Combining this with the result of the first paragraph established the theorem.

The strictness, $I_{2}<0$, in the interior comes from a further application of Maximum Principle 2.2.

Since translates of logconcave functions are not necessarily logconcave, the following cannot obviously be deduced from Theorem 1.0.

THEOREM 4.1. Let $u$ solve

$$
\begin{gathered}
u_{t}=u_{x x} \\
u( \pm a, t)=M>0, \\
u(x, 0)=u_{0}(x) \geq M>0, \quad u_{0}( \pm a)=M,
\end{gathered}
$$

with $u_{0}$ logconcave.

Then $u(\cdot, t)$ is logconcave at all subsequent times. 
Proof. The results of Section 3 can be extended to cover the changed Dirichlet conditions. For example an informal description of the extension of Lemma 3.5 is as follows. Since

$$
u(x, t) \sim M+c \exp \left(-\frac{\pi^{2} t}{4 a^{2}}\right) \cos \left(\frac{\pi x}{2 a}\right),
$$

with

$$
c=\frac{1}{a} \int_{-a}^{a}\left(u_{0}(x)-M\right) \cos \frac{\pi x}{2 a} d x>0,
$$

the function $u(\cdot, t)$ is concave at large time and

$$
I_{2}\left(M+c u_{1}\right)=M c u_{1 x x}+c^{2} I_{2}\left(u_{1}\right)<0,
$$

for $M>0$ and $c>0$.

After noting that we still have

$$
I_{2}( \pm a)=-u_{x}( \pm a)^{2}<0,
$$

the proof is identical to that of Theorem 1.0.

\section{Logconvexity in $t$}

Define

$$
J_{2}=u u_{t t}-u_{t}^{2}
$$

Observe that, when $u$ satisfies the heat equation, $J_{2}=\left(I_{2}\right)_{x x}$.

Theorem 5.0. Let $\Omega=(-a, a) \subset \mathbf{R}$. Suppose that $u$ satisfies the heat equation, is positive and is sufficiently smooth. Suppose that $J_{2} \geq 0$

(i) initially, that is for $t=0$, and

(ii) in neighbourhoods of the boundaries $x= \pm a$.

Then, at fixed values of $x$, the distribution of temperature $u(x, t)$ is a logconvex function of $t$.

REMARK. The function $\left(M+u_{1}\right)$ for $M \geq 0$ satisfies the hypotheses of Theorem 5.0 and for it $J_{2} \geq 0$ everywhere, the case where $M$ is zero corresponding to $J_{2}$ being zero everywhere.

Proof. A calculation gives

$$
\left(I_{2 x}\right)_{t}-\left(I_{2 x}\right)_{x x}+\frac{2 u_{x}}{u}\left(I_{2 x}\right)_{x}=\frac{2 u_{t}}{u}\left(I_{2 x}\right)
$$

and hence

$$
J_{2 t}-J_{2 x x}+\frac{2 u_{x}}{u} J_{2 x}-\frac{2 u_{x}^{2}}{u^{2}} J_{2}=\frac{2}{u^{2}} I_{2 x}^{2}
$$


Also $J_{2} \geq 0$ at, and in from, the boundary and initially. Using this an application of the Maximum Principle 2.2 establishes the theorem.

Remark. Consider positive solutions of problem (P). Because the coefficients in equation (5.1) become unbounded as $x$ tends to $\pm a$, merely knowing that $J_{2} \geq 0$ on the parabolic boundary $S_{T}$ is not good enough to establish that $J_{2} \geq 0$ in $Q_{T}$. For all positive solutions of problem (P), $J_{2}( \pm a)=0=J_{2 x}( \pm a)$ while

$$
J_{2 x x}( \pm a)=2\left(u_{x}( \pm a) u_{x t t}( \pm a)-u_{x t}( \pm a)^{2}\right) .
$$

With the additional hypothesis that $J_{2} \geq 0$ initially, the first of the two terms on the right hand side is positive. (This is because $J_{2} \geq 0$ implies that $u_{t t} \geq 0$, and with this known around the boundary the Maximum Principle establishes that $u_{t t}>0$ in the interior, and $u_{x}( \pm a) u_{x t t}( \pm a)>0$.) Necessary for hypothesis (ii), for problem (P), is that $J_{2 x x} \geq 0$.

Consideration of the asymptotics at large time indicates that very few positive solutions of problem (P) will have $J_{2} \geq 0$ on $\Omega \times[0, \infty)$.

\section{Concavity properties jointly in $(x, t)$}

6.1. Logconcavity jointly in $(x, t)$. Kennington [15] has established, by different techniques, for problems similar to ours, various results concerning concavity jointly in $(x, t)$.

Consider positive functions of the two variables $(x, t)$. When these are suitably smooth these are jointly concave (in $(x, t)$ ) if the matrix of second partial derivatives is negative semidefinite. A positive function $u(x, t)$, which is suitably smooth, is jointly logconcave if and only if

$$
\begin{gathered}
I_{2}=u u_{x x}-u_{x}^{2} \leq 0, \quad J_{2}=u u_{t t}-u_{t}^{2} \leq 0, \\
K_{2}=\left(u u_{x x}-u_{x}^{2}\right)\left(u u_{t t}-u_{t}^{2}\right)-\left(u u_{x t}-u_{x} u_{t}\right)^{2} \geq 0 .
\end{gathered}
$$

The final condition immediately above can be written

$$
K_{2} / u=u H+T \geq 0,
$$

where

$$
\begin{gathered}
H=\left(u_{x x} u_{t t}-u_{x t}^{2}\right), \\
T=\left(-u_{t}^{2} u_{x x}+2 u_{x} u_{t} u_{x t}-u_{x}^{2} u_{t t}\right) .
\end{gathered}
$$

We remark that $I_{2} \leq 0$ and $K_{2} \geq 0$ implies that $J_{2} \leq 0$.

Although the function $u_{1}$ satisfies the hypotheses, Theorem 6.0 is unlikely to be useful for problem $(\mathrm{P})$. Outside the context of the zero Dirichlet conditions of problem $(\mathrm{P})$, there are other positive solutions of the heat equation, which have joint concavity properties. 
THEOREM 6.0 (Interior joint concavity maximum principle). Let $\Omega=$ $(-a, a) \subset \mathbf{R}$. Suppose that $u$ solves the heat equation, is positive, and is sufficiently smooth. Suppose that (a) $I_{2}<0$ in $Q_{T}$. Suppose also that $K_{2}$ is nonnegative

(i) initially, that is for $t=0$, and

(ii) in neighbourhoods of the boundaries $x= \pm a$.

Then $K_{2} \geq 0$ everywhere and, using (a), the distribution of temperature $u(\cdot, \cdot)$ is a logconcave function of $(x, t)$.

REMARK. A similar joint-logconvexity maximum principle is obtained when condition (a) above is replaced by (b) $I_{2}>0$ in $Q_{T}$.

Proof. Our proof consists in verifying that $K_{2}$ satisfies a parabolic equation,

$$
K_{2 t}-K_{2 x x}+A_{1} K_{2 x}+A_{0} K_{2}=0
$$

with

$$
A_{1}=C_{1} /\left(u I_{2}\right), \quad A_{0}=-C_{0} /\left(u^{2} I_{2}\right),
$$

where

$$
C_{1}=2\left(u I_{2}\right)_{x}, \quad C_{0}=2\left(-u_{x}^{4}+u^{3} u_{t t}\right) .
$$

The behaviour of the coefficients is such that the Maximum Principle 2.2 can be applied to find that the property $K_{2} \geq 0$ persists if it is true initially.

(Though not used in proofs in this paper the identities of this paragraph may be of interest and of use in settling the questions given in Section 6.2. We have

$$
K_{2}=u^{4} \operatorname{det}\left(\begin{array}{cc}
(\log u)_{x x} & (\log u)_{x t} \\
(\log u)_{x t} & (\log u)_{t t}
\end{array}\right)
$$

Using that $u$ solves the heat equation we have

$$
\begin{aligned}
K_{2} & =\operatorname{det}\left(\begin{array}{cc}
\left(u u_{t}-u_{x}^{2}\right) & \left(u u_{x t}-u_{x} u_{t}\right) \\
\left(u u_{x t}-u_{x} u_{t}\right) & \left(u u_{t t}-u_{t}^{2}\right)
\end{array}\right), \\
& =\operatorname{det}\left(\begin{array}{cc}
I_{2} & I_{2 x} \\
I_{2 x} & I_{2 x x}
\end{array}\right)=I_{2}^{2} \frac{\partial^{2}}{\partial x^{2}} \log \left(\left|I_{2}\right|\right) .
\end{aligned}
$$

Thus a solution $u$ of the heat equation is jointly logconcave if and only if $\left(-I_{2}\right)$ is a nonnegative function which is logconvex in $x$ at each fixed value of $t$. Another identity is that

$$
K_{2} / u=\operatorname{det}\left(\begin{array}{ccc}
u_{x x x x} & u_{x x x} & u_{x x} \\
u_{x x x} & u_{x x} & u_{x} \\
u_{x x} & u_{x} & u
\end{array}\right)
$$


Thus $K_{2} / u$ is a Hankel determinant of order three, as defined in $[9$, p. 70], while $I_{2}$ is a Hankel determinant of order two.)

Consider problem (P). Using the asymptotic expressions (1.1a) and (1.1b), it can be verified that, for sufficiently large time, each of $-I_{2}$ and $T$ are positive. Also $-J_{2}$ and $K_{2}$ tend to zero faster than $u^{2}$. In particular the solution given by the right-hand side of $(1.1 \mathrm{a})$, that is $(1.1 \mathrm{c})$, is jointly logconcave. More generally consider the problem in Theorem 4.1. Since the boundary data is independent of time $u_{t}( \pm a)=0$ so that $J_{2}( \pm a)=0$ and so $K_{2}( \pm a)=-u( \pm a)^{2} u_{x t}( \pm a)^{2} \leq 0$. Thus for such problems it will not be common for the solution to be jointly logconcave.

6.2. Other joint concavity questions for problem $(P)$. The kernel function $K$ for the heat equation is logconcave in $x$. It is not jointly logconcave everywhere in $(x, t)$. (An explicit calculation of $J_{2}$ for the infinite-interval kernel function $K_{\infty}$ shows that $J_{2}$ can take on either sign depending on where in $(x, t)$ it is evaluated. Similarly an explicit calculation of $K_{2}$ for the infiniteinterval kernel function $K_{\infty}$ shows that then $K_{2}$ is negative for all $(x, t)$.)

To see that $K$ is not jointly logconcave everywhere in $(x, t)$, consider asymptotics at large time. Recall that $K_{2}\left(u_{1}\right)=0$. Also, for solutions of problem $(\mathrm{P}), K_{2}$ is zero on $x= \pm a$. We have

$$
\begin{aligned}
K(x, \hat{x}, t) \sim & \exp \left(-\frac{\pi^{2} t}{4 a^{2}}\right) \cos \left(\frac{\pi \hat{x}}{2 a}\right) \cos \left(\frac{\pi x}{2 a}\right) \\
& +\exp \left(-\frac{\pi^{2} t}{a^{2}}\right) \sin \left(\frac{\pi \hat{x}}{a}\right) \sin \left(\frac{\pi x}{a}\right) .
\end{aligned}
$$

From this a calculation establishes that the sign of $K_{2}(K)$ at large time depends on $x$ and on $\hat{x}$.

Although not jointly logconcave in $(x, t)$, the kernel function for the heat equation may, however, be jointly quasiconcave in $(x, t)$. Evidence for this is that, for the function $K_{\infty}$, this property can be easily verified.

Question 6.1. Let $\Omega=(a, b) \subset \mathbf{R}$ and let $K$ be the kernel function for the heat equation, with zero data at the endpoints of the interval. Let

$$
T(K)=\left(-K^{\prime \prime}\right)^{3}+2 K^{\prime} K^{\prime \prime} K^{\prime \prime \prime}-\left(K^{\prime}\right)^{2} K^{\prime \prime \prime \prime} \text {. }
$$

Is the distribution of temperature $K(x, \hat{x}, t)$ a quasiconcave function of $(x, t)$, that is, is $T(K)>0$ for all time?

Evidence in favour of a positive answer to Question 6.1 is that, not only is the result true when $\Omega=\mathbf{R}$, but the result is also true when $a=0$ and $b=\infty$. In this latter case the kernel $K_{S}$ is given by

$$
K_{S}(x, \hat{x}, t)=K_{\infty}(x, \hat{x}, t)-K_{\infty}(x,-\hat{x}, t) .
$$


The REDUCE programs used to check that $T\left(K_{\infty}\right)>0$ and $T\left(K_{S}\right)>0$ are given in Keady [13]. (We also have, for the finite interval $(-a, a)$, numerical evidence in favour of a positive answer to Question 6.1.)

Many identities related to attempts to answer the questions in this section are given in [13]. One is that

$$
T+u H=\frac{K_{2}}{u}=-\frac{I_{2} T}{u_{x}^{2}}-\frac{u\left(u_{t}^{2}-u_{x} u_{x t}\right)^{2}}{u_{x}^{2}} .
$$

From the right hand equality of (6.2) it is immediate that, for solutions with $I_{2} \leq 0, T \geq 0$ wherever $K_{2} \geq 0$.

Since $K_{2} / u=T+u H$, at points where $K_{2} \leq 0$ and $T \geq 0$ we must have $H \leq 0$. Also from equations (6.2) at points where $u_{t} T \geq 0$ we have $H \leq 0$. More might be true. We have that $H(K)<0$ for sufficiently large values of time.

QUESTION 6.2. Is $H(K)<0$ everywhere?

We remark that

$$
H_{t}-H_{x x}+\frac{2 u_{x t}}{u_{t}} H_{x}-\frac{2 u_{t t}}{u_{t}} H=0,
$$

at points where $u_{t}=0, H=-u_{x t}^{2} \leq 0$, and at $x= \pm a, H \leq 0$. This suggests that if $H \leq 0$ initially it is likely that $H$ will stay nonpositive.

Several related questions are discussed in [13].

\section{References}

[1] A. Acker, L. E. Payne and G. Philippin, 'On the convexity of level lines of the fundamental mode in the clamped membrane problem, and the existence of convex solutions in a related free boundary problem', Z. Angew. Math. Phys. 32 (1981), 683-694.

[2] P. Benilan and J. L. Vazquez, 'Concavity of solutions of the porous medium equation', Trans. Amer. Math. Soc. 299 (1987), 81-93.

[3] H. J. Brascamp and E. H. Lieb, 'On extensions of the Brunn Minkowski and Prekopa Liendler inequalities, including inequalities for logconcave functions, and with an application to the diffusion equation', J. Functional Anal. 22 (1976), 366-389.

[4] L. A. Caffarelli and A. Friedman, 'Convexity of solutions of semilinear elliptic equations', Duke Math. J. 52 (1985), 431-456.

[5] R. S. Ellis and C. M. Newman, 'Extensions of the Maximum Principle: exponential preservation by the heat equation', J. Differential Equations 30 (1978), 365-379.

[6] H. Engler, 'Contractive properties for the heat equation in Sobolev spaces', J. Funct. Anal. 64 (1985), 412-435.

[7] A. Friedman, Partial differential equations of parabolic type (Prentice-Hall, 1964).

[8] B. Gidas, W. M. Ni and L. Nirenberg, 'Symmetry and related problems via the Maximum Principle', Comm. Math. Phys. 68 (1979), 209-243. 
[9] S. Karlin, Total positivity (Stanford University Press, 1968).

[10] B. Kawohl, Rearrangements and convexity of level sets in P.D.E., Lecture Notes in Math., vol. 1150 (Springer, Berlin, 1985).

[11] B. Kawohl, 'Qualitative properties of solutions to semilinear heat equations', Expositiones Math. 4 (1986), 257-270.

[12] G. Keady, 'The power concavity of solutions of some semilinear elliptic boundary-value problems', Bull. Austral. Math. Soc. 31 (1985), 181-184.

[13] G. Keady, The persistence of log-concavity for positive solutions of the one-dimensional heat equation, Res. Rep. Math. Dept. Univ. of Western Australia (February 1987). With corrections, January 1988.

[14] G. Keady and I. Stakgold, Combustion of convex solids, Res. Rep. Math. Dept. Univ. of Western Australia (May 1987).

[15] A. U. Kennington, 'Concavity of level curves for an initial value problem', J. Math. Anal. Appl. 133 (1988), 324-330.

[16] N. Korevaar, 'Convex solutions to nonlinear elliptic and parabolic boundary value problems', Indiana Univ. Math. J. 32 (1983), 603-614.

[17] L. G. Makar-Limanov, 'Solution of Dirichlet's problem for the torsion equation in a convex region', Math. Notes Acad. Sci. U.S.S.R. 9 (1971), 52-53.

[18] H. Matano, 'Nonincrease in the lap number for a one-dimensional semilinear parabolic equation', J. Fac. Sci. Univ. Tokyo IA 29 (1982), 401-441.

[19] K. Nickel, 'Gestaltaussagen über Losungen parabolischer Differentialgleichungen', J. Reine Angew. Math. 211 (1962), 78-94.

[20] G. Polya, 'Qualitatives der warmeausgleich', Z. Angew. Math. Mech. 13 (1933), 125-128.

[21] M. H. Protter and H. F. Weinberger, Maximum principles in differential equations (Prentice-Hall, Englewood Cliffs, N.J., 1967).

[22] R. Sperb, Maximum principles and their applications (Academic Press, New York, 1981).

\section{Mathematics Department}

University of Western Australia

Nedlands, W. A. 6009

Australia 